\title{
Multiphysics \& Parallel Kinematics Modeling of a 3DOF MEMS Mirror
}

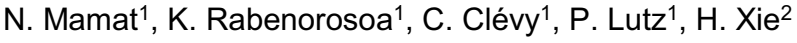 \\ ${ }^{1}$ FEMTO-ST Institute, AS2M department, Univ. Bourgogne Franche-Comté, Univ. de Franche-Comté CNRS/ENSMM, 24 \\ rue Alain Savary, 25000 Besançon, France \\ ${ }^{2}$ Interdisciplinary Microsystems Group, University of Florida, Gainesville, USA
}

\begin{abstract}
This paper presents a modeling for a 3DoF electrothermal actuated micro-electro-mechanical (MEMS) mirror used to achieve scanning for optical coherence tomography (OCT) imaging. The device is integrated into an OCT endoscopic probe, it is desired that the optical scanner have small footprint for minimum invasiveness, large and flat optical aperture for large scanning range, low driving voltage and low power consumption for safety reason. With a footprint of $2 \mathrm{~mm} \times 2 \mathrm{~mm}$, the MEMS scanner which is also called as Tip-Tilt-Piston micromirror, can perform two rotations around $\mathrm{x}$ and $\mathrm{y}$-axis and a vertical translation along $\mathrm{z}$-axis. This work develops a complete model and experimental characterization. The modeling is divided into two parts: multiphysics characterization of the actuators and parallel kinematics studies of the overall system. With proper experimental procedures, we are able to validate the model via Visual Servoing Platform (ViSP). The results give a detailed overview on the performance of the mirror platform while varying the applied voltage at a stable working frequency. The paper also presents a discussion on the MEMS control system based on several scanning trajectories.
\end{abstract}

\section{INTRODUCTION}

The applications of MEMS mirror can be found in numerous optical systems [1]. In medical research [2], MEMS mirror have been used in OCT imaging for transverse scanning [3], [4]. Most OCT probes are based on MEMS scanners of out-of-plane motion type [5], [6], [7], [8].

In literature, we can find different kinds of mirror actuation mechanisms such as piezoelectric, electrostatic, electromagnetic and electrothermal [9]. The MEMS mirror studied used electrothermal actuations which consume high power and work at low speeds, compared to the others actuation mechanism. But the advantage lies in large scan ranges at low driving voltages, making them ideal for OCT imaging where the operation is at low speed and doesn't consume much energy. Several designs with electrothermal actuation have been presented [10], [11], [12], [13], with different number of DoF and diverse actuator's design. Most of them have been demonstrated by the University of Florida research team led by the professor Huikai Xie. One of the research works involves in making biomedical imaging device.

Various characterization and modeling works have been carried out but most of them focused on design and optimization. MEMS characterization and modeling is a very complex task [14], as it requires adequate experimental procedures and sophisticated tools for handling the device properly. Few works can be found about the procedure in literature [15].

The MEMS mirror is relatively small and the actuators have to deflect to perform out-of-plane scan. Because of its small size, integrating sensors is quite problematic. The large deflections along with the active materials as the actuation mechanism influence the behavior of the MEMS mirror which caused the nonlinearity. Hence, it is required to construct a fine model of each elementary actuator and the overall system.

We present a novel approach of combining the multiphysics characterization with the parallel kinematics modeling based on parallel robots. The objective is to correctly predict the behavior of the actuators and also to be able to control them afterwards. The MEMS scanner might be used as a parallel micromanipulator later. We can find some works on micromanipulators in medical application [16].

In this paper, the MEMS mirror is presented in section II. Section III describes the modeling of 3DoF MEMS mirror based on multiphysics characteristics and parallel kinematics. Section IV discloses the experimental validation followed by a discussion on a control system in section V. Finally, the section VI provides some conclusions. 


\section{PRESENTATION OF MEMS MIRROR}

The overall system includes a mobile platform of the micro-mirror which is attached to a fixed substrate via four electrothermal actuators that can be controlled together or separately. This configuration allows 3 DoF motions: two rotations and a vertical translation. The device footprint is about $2 \mathrm{~mm} \times 2 \mathrm{~mm}$ whereas the mirror platform is $1 \mathrm{~mm} \times 1 \mathrm{~mm}$. The actuation mechanism used for the micromirror is the Inverted-Series-Connected (ISC) bimorph design.

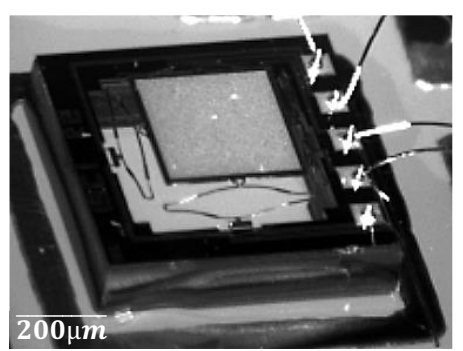

Figure 1. The side view of the MEMS mirror

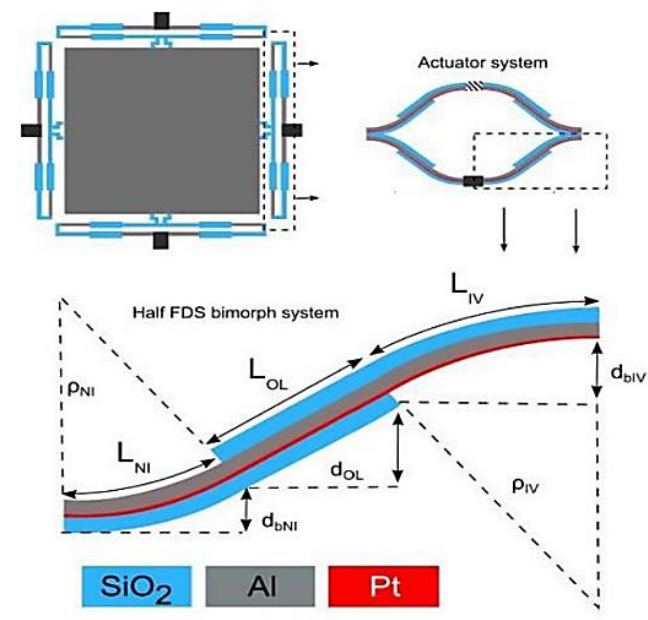

Figure 2. Top left: Position of actuators in the MEMS system. Top right: Structure of an electrothermal actuator. Bottom: A single bimorph system with details on different sections and the materials used $\lceil 17\rceil$.

Each actuator consists of four Folded Dual S-Shaped (FDS) bimorphs which assure a pure lateral displacement of the mirror platform during piston or tip-tilt motion. At room temperature i.e. when no voltage is applied, the FDS bimorph is at an initial elevated position. When the voltage is applied, the FDS bimorph is deflected and moves downwards, the mirror platform shifts towards the fixed substrate. A half of the FDS bimorph is called an S-shaped.

Each S-shaped bimorph consists of 3 sections: the inverted sections (IV), the non-inverted sections (NI) and the overlap sections (OL). Both inverted and non-inverted parts are not symmetrical. It is due to the fabrication process to assure the electrical isolation between the two layers of $\mathrm{Al}$ and $\mathrm{Pt}$ via a layer of $\mathrm{SiO} 2$. The fabrication and optimization procedures of the ISC bimorph and the micro mirror can be found in [11] and [17].

\section{MEMS MIRROR MODELING}

Given the drive voltages as the main inputs which lead to the displacement of each actuator, we shall obtain two tilting angles $\theta, \beta$ and a vertical displacement $p z$ as the main outputs. (See Fig. 3)

In the first part, we studied the behavior of the MEMS mirror in response to external stimuli. Then, we studied the parallel kinematics aspect of the MEMS which is to predict the position of the mirror platform when the length of the actuators varied.

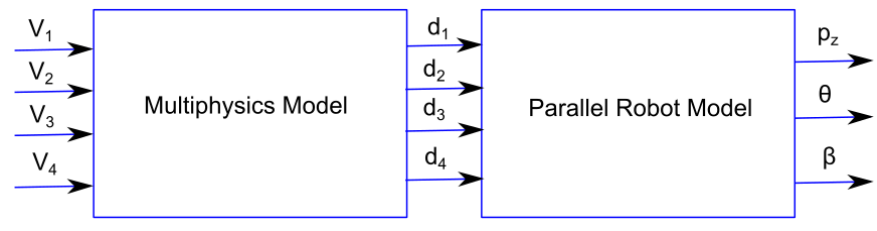

Figure 3 Block diagram of the modeled system.

\subsection{Multiphysics modeling}

Multiphysics modeling approach is chosen based on several physics aspects which describe or influence the behavior of the microsystem:

- Electrical: Applied voltages as the input variables

- Mechanical: The actuator is modeled as a fixedpinned cantilever beam, the actuation forces, the materials used in the actuators' fabrication process influence their torque and stiffness

- Thermodynamics: The temperature change effects their electrothermal cycle

- Energy: The actuators are considered as an energy transducer.

\subsubsection{Actuator's displacement during the piston motion}

The modeling of the electrothermal actuated mirror is based on Espinosa et al.'s work [17]. The preliminary work modelled the actuator as a simple cantilever beam and presented the analytical model of the actuator.

We observed that modeling the actuator as a simple cantilever beam denies the existence of different physical interactions which caused the actuation forces. Therefore, in the following work, the actuator is modelled as a fixedpinned cantilever beam. It is proposed based on several reasons. The shape of the cantilever resembles the shape of the S-Shaped bimorph. Studying the S-shaped as a whole instead of focusing on each sections (IV, NI, OL) minimize the uncertainty caused by the asymmetry between these sections. We are also concerned by the force generated to withstand the actuator's displacement. This force is due to the reaction between the mirror platform and the actuator's stiffness. 


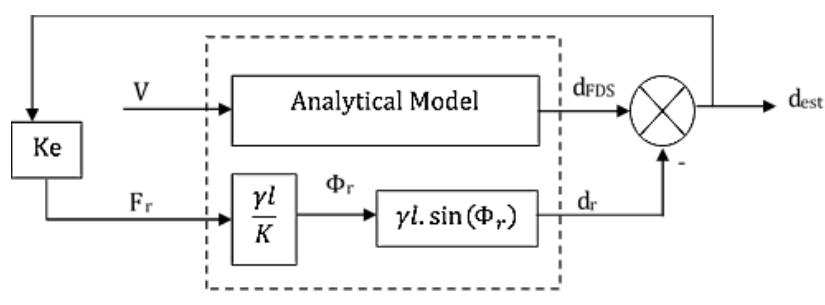

Figure 4 Blocks diagram to estimate FDS actuator vertical displacement as a fixed-pinned cantilever beam

The figure above shows the diagram of the model based on a fixed-pinned cantilever beam where $l$ are the length of the beam, $F_{r}$ is the applied vertical force which deflects the beam, $\gamma$ is the characteristic radius factor, and $\Phi_{r}$ is the pseudo-rigid-body angle. Given the variables, we obtain the equation of the pseudo-rigid-body angle:

$$
\Phi_{r}=\frac{\gamma l}{K} F_{r}
$$

Where $\gamma=0.8517, l=500 \mu m$ and $K$ is the S-Shaped torsional constant.

The constant $K$ is calculated based on the equation:

$$
K=2 \gamma K_{\theta} \frac{E . I}{l}
$$

Where $K_{\theta}$ is the beam's stiffness coefficient, $E$ is the beam's Young's modulus and I is the area moment of inertia. As stated before, an actuator consists of four Sshaped bimorphs. In the first place, we focus on one Sshaped and we contemplate that the other three as its external mechanisms.

$K_{e}$ is the pulling stiffness which would be identified experimentally.

$$
K_{e}=\frac{d_{e s t}}{F_{r}}
$$

The estimated displacement $d_{e s t}$ is the result of the deflection of an S-shaped minus the displacement caused by vertical actuation force.

\subsubsection{Displacement during the tip-tilt motion}

The behavior of the S-shaped of each actuator is defined separately. We take into consideration the torsion stiffness introduced by the tip-tilt motion. For example, if the tilting is around axis formed by actuators 2 and 4, they will undergo a torque stress at the extremities where they are connected to the mirror plate as a result of the actuators' alternating motions of going up and down. The tilting angle $\alpha$ is the angle between the referential position of mirror platform and its new position after tilting, as illustrated in Fig. 9. We use the position of mirror plate when $2 \mathrm{~V}$ voltage is applied to all actuators as the reference.

An angular moment $M_{\text {rot }}$ is generated on the actuators which formed the rotational axis.

$$
M_{\text {rot }}=\alpha K_{\text {rot }}
$$

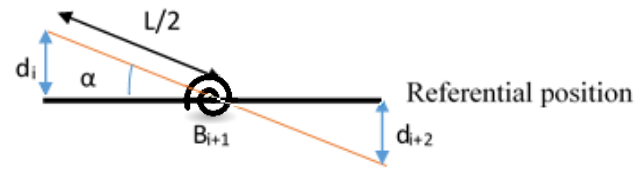

Figure 5 Both actuators' displacements are assumed to be purely vertical during tip-tilting motion.

$K_{\text {rot }}$ is the angular stiffness of the mirror-actuator joint. Then we deduce the rotational force $F_{\text {rot }}$.

$$
F_{\text {rot }}=\frac{2}{L} M_{\text {rot }}
$$

This force will be added to the applied vertical actuation force $F r$. The estimated displacement of each actuator depends on the angle of rotation.

$$
d_{e s t}=\frac{F_{r}+F_{r o t}}{K_{e}}=\frac{F_{r}+\frac{2}{L}\left(\alpha K_{r o t}\right)}{K_{e}}
$$

We come up with some working hypotheses:

1. The actuators displacement is purely vertical during rotation. (as shown in Fig. 5)

2. There's only a pair of actuator triggered during a rotation around one axis.

3. The actuators are all identical.

4. The applied vertical actuation force $F r$ is considered equivalent for all the $\mathrm{S}$-shaped of the actuator.

5. Angular stiffness for rotation around both axis is defined separately and their values are estimated from the single angular stiffness Krot. This step is important because the $3 \mathrm{DoF}$ may introduce additional constraints than in 1 DoF and 2 DoF motions. Both constants shall be identified experimentally.

\subsection{Geometrical model based on parallel robot}

The MEMS mirror is modelled as a parallel robot, based on the Stewart-Gough (SP) platform. Two reference frames are assigned; the first one is the absolute frame $R s=(O s, x, y, z)$ tied to the fixed base (see Fig. 6) and the second one is the mobile frame $R p=(O p, u, v, w)$ tied to the mobile mirror platform.

The extremities of each leg are fitted with a 3 DoF spherical joint at the base and at the platform. The spherical joint centers are denoted by $A i$ and $B i(i=1, . ., 4)$. The length of each leg/actuator are denoted by $\operatorname{di}(i=1, . ., 4)$.

- Let $O s$ be the origin of the absolute coordinate system.

- Let $O p$ be the origin of the mobile frame $R p$.

- $A i$ is the center of the joint at the base.

$$
A_{i}=\left[\begin{array}{lll}
a_{i}^{x} & a_{i}^{y} & a_{i}^{z}
\end{array}\right]
$$

- $B i$ is the center of the joint at the mobile platform.

$$
B_{i}=\left[b_{i}^{x} b_{i}^{y} b_{i}^{z}\right]
$$




\section{MATEC Web of Conferences}

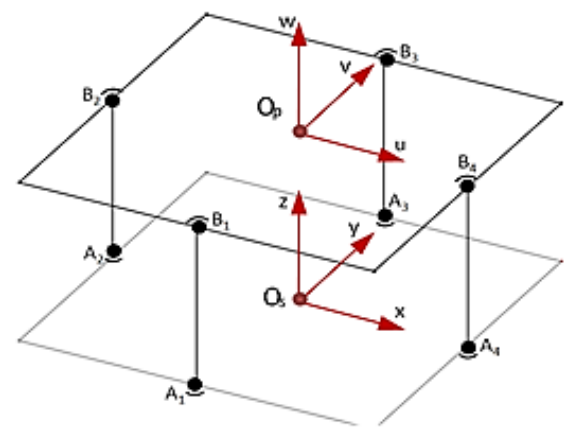

Figure 6 Geometrical representation of the MEMS platform as a parallel robot with four legs/actuators

The coordinates of $A i$ and $B i$ are defined in the absolute frame $R s$ hence $b_{i}^{z}=d_{i}$.

- $\quad d i$ is the scalar product of the vector $\overrightarrow{A_{l} B_{l}}$ and is given by:

$$
\left\|d_{i}\right\|=\overline{A_{l} B_{l}}=p+R b_{i}-a_{i}
$$

- $[R]$ is the $3 \times 3$ rotation matrix of $r_{i j}$ elements, expressing the orientation of the $R p$ mobile frame with respect to the $R s$ absolute frame.

- $\theta$ and $\beta$ are the angles describing the rotation of the mobile platform with respect to the fixed base.

- $\vec{p}=\left[\begin{array}{lll}0 & 0 & p_{z}\end{array}\right]$ is the $\mathrm{z}$-axis translation vector of the mobile platform. We defined $p z$ as the vertical translation of the mobile platform center.

- $\vec{u}=\left[\begin{array}{lll}u_{x} & u_{y} & u_{z}\end{array}\right]^{t}=\left[\begin{array}{lll}\cos \beta & 0 & \sin \theta\end{array}\right]^{t}$ is a unit vector which passed through $O p$. (see Fig.7)

- $\vec{v}=\left[\begin{array}{lll}v_{x} & v_{y} & v_{z}\end{array}\right]^{t}=\left[\begin{array}{lll}0 & \cos \theta & \sin \theta\end{array}\right]^{t}$ is a unit vector which passed through $O p$. (see Fig. 8)

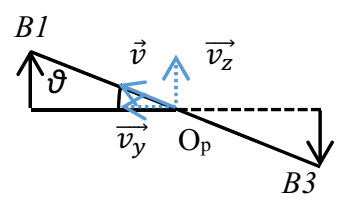

Figure 8 Detail on unit vector $\mathrm{v}$ in $\mathrm{y}-\mathrm{z}$ plane during a rotation around $\mathrm{x}$-axis

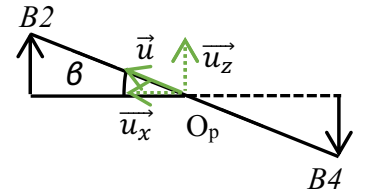

Figure 7 Detail on unit vector $\mathrm{u}$ in $\mathrm{x}-\mathrm{z}$ plane during a rotation around $y$-axis
We come up with some working hypotheses so as to construct the robotic model:

1. The four joints at the mobile platform are in the $u$-v plane therefore the $w$ component of $R p$ is null.

2. The four joints at the fixed base are in the $x-y$ plane therefore the $z$ component of $R s$ is null.

3. The mobile platform lateral shifting in $u$ - $v$ plane is nonnegligible during rotations and thus would be taken into consideration when solving the model system of equations.

4. $p z$ would be the average of the length of each leg $d_{i}$.

$$
p_{z}=1 / 4 \times \sum d_{i}
$$

Based on these hypotheses, we formulated the general transformation matrix of $R s$ to $R p$ as ${ }^{S} T_{P}$.

\subsubsection{DoF model}

Contrarily to the 2 DoF model, rotation angles $\theta$ and $\beta$ are dependent to each other and would have definite values. To simplify the task, we emphasize the 3 DoF model as a vertical translation $p z$ followed by a tip-tilting around a first axis then ensued by a second tip-tilting around a second axis. Hence, the general transformation matrix of $R s$ to $R p$ is defined as

$$
{ }^{S} T_{P}=T_{p, \theta \mid I} \cdot T_{\beta \mid I I}
$$

The first axis $I$ could be either $\mathrm{x}$ or $\mathrm{y}$ at a time and the second axis $I I$ could be $\mathrm{u}$ or $\mathrm{v}$, depending on the previous axis used. $T_{p, \theta \mid I}$ is a transformation matrix which combines the translation and a rotation around the first axis.

$T_{\beta \mid I I}$ is the second transformation matrix defined by a second rotation by an angle of $\beta$ about an axis in the direction of a unit vector $\vec{v}$ or $\vec{u}$.

We combined both systems of equations of the $2 \mathrm{DoF}$ model and deduce the values of the angles $\theta$ and $\beta$ from there. We used the Eq. (1) and the elements $r_{i j}$ to identify the three main 3 DoF model variables; the two rotation angles $\theta, \beta$ and the vertical translation $p z$.

\section{EXPERIMENTAL RESULTS}

The objective of this section is to validate the modeling presented before.

\subsection{Experimental setup}

The geometrical configuration of the experimental setup is shown in Fig. 9. The laser points at the center of the micromirror and the laser spot movement is captured by a CCD camera. The data containing the coordinates of the laser spot is then processed in Simulink ${ }^{\circledR}$. Working range has been set from 0 to $3.5 \mathrm{~V}$. When the applied voltage exceeds $3.5 \mathrm{~V}$, the actuators began to lose their elasticity.

The mirror platform displacements during the piston motion are considered as relative displacements. The initial position of the mirror at $0 \mathrm{~V}$ is about $220 \mu \mathrm{m}$ above the substrate. The positive $\mathrm{z}$-axis translation is toward the substrate. At $0 \mathrm{~V}$, the displacement corresponds to $0 \mu \mathrm{m}$. The four actuators are applied with a constant voltage of 0 to $3.5 \mathrm{~V}$ with $0.5 \mathrm{~V}$ variance. Next, we applied $2 \mathrm{~V}$ to all actuators plus an alternative component $\Delta V$ which is applied on two opposite actuators. We set the value $\Delta V=0.5 \times \sin (2 \pi f+\varphi)$ with $f=0.5 \mathrm{~Hz}$ and $\varphi=[0, \pi]$. Hence, the voltage applied onto these two actuators varies between 1.5 to $2.5 \mathrm{~V}$.

The displacement is calculated from the reference position of the mirror at $2 \mathrm{~V}$. Finally, for validating the 3 DoF, we choose the maximum angle obtained by tip-tilt motion around one axis as the reference angle. 


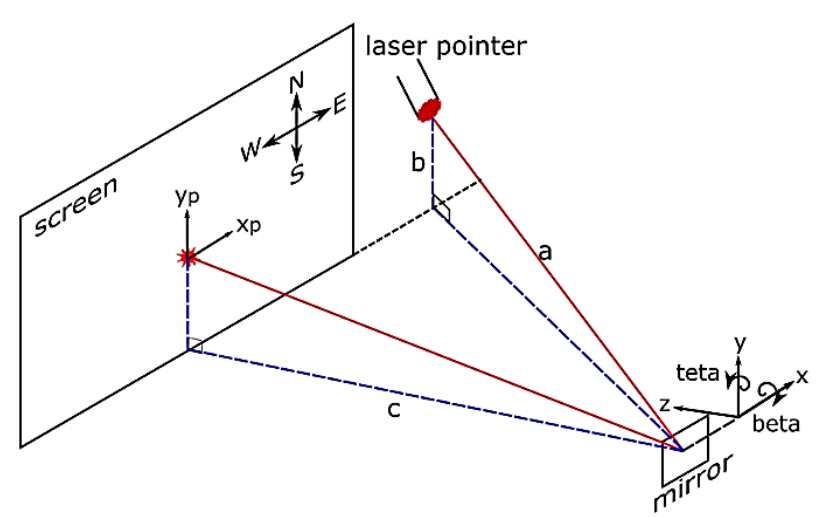

Figure 9 Geometrical configuration of the experimental setup

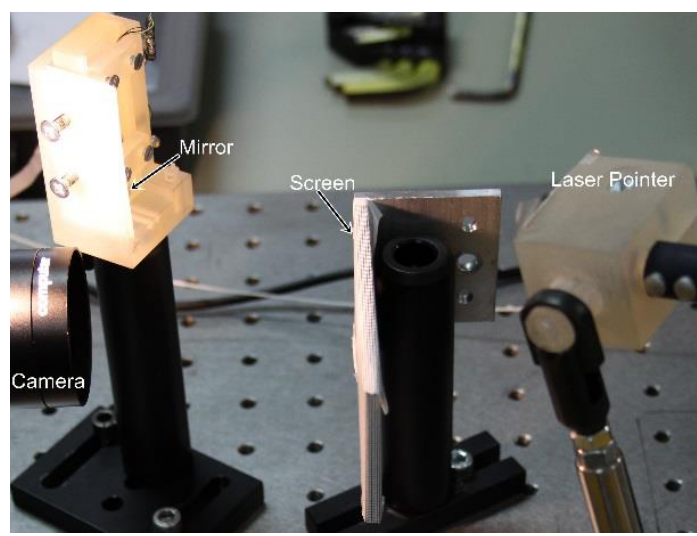

Figure 10 Schematic of experimental setup

\subsection{Identification of $\mathrm{Ke}$ and $\mathrm{Krot}$}

The pulling stiffness and the angular stiffness are difficult to calculate due to the uncertainties on the physical parameters. We propose to identify both constants based on measurement displacement for piston motion and tiptilt motion with the parameter values of the electrothermal actuators given. The computation gives $K e=58.216 \mathrm{~N} / \mathrm{m}$ and $K r o t=4.99 \times 10^{-5} \mathrm{Nm} / \mathrm{rad}$ which will be used for the model validation. The value of Krot is also used for the 3 DoF model validation.

\subsection{Model validation}

\subsubsection{Piston motion}

Fig. 11 shows the comparison between the model and the experimental result and also the displacement error. The maximum displacement obtained is $173.6 \mu \mathrm{m}$ at $3.5 \mathrm{~V}$.

The difference between both curves may be due to the uncertainties in the dimensions of bimorph's different layers of materials.

We concluded that the error between the experimental and the model results may be caused by the submitted working hypotheses defined during modeling process. We observe that the different sections of the bimorph are not symmetrical and the fault in the fabrication process are non-negligible.

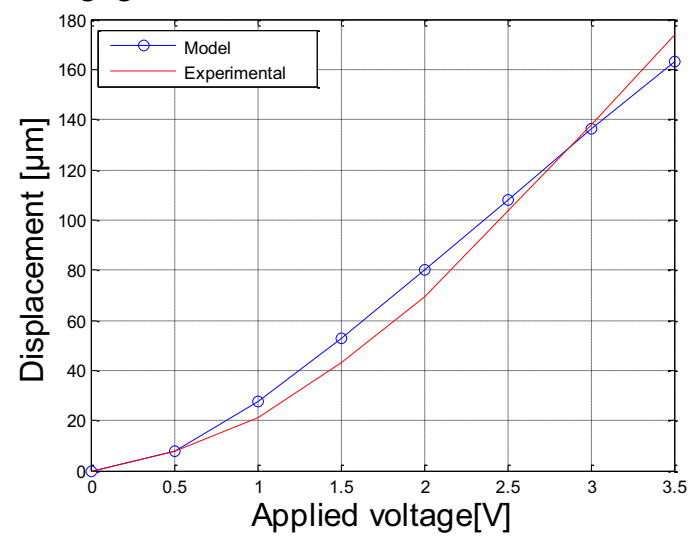

Figure 11 Comparison between model \& experimental data for piston motion

\subsubsection{DOF Piston-Tip-Tilt motion around one axis at each time}

In Fig. 12, we can see that the both curves are more or less identical to model data. The maximum angle for rotation around $\mathrm{x}$-axis achieved is $\theta=1.463^{\circ}$.

We switched the tip-tilt axis to see if the other actuators 2 and 4 behave the same as the actuator 1 and 3 during tiptilt motion around one axis.

Krot is proven to be the same as in previous test. However, the maximum angle obtained for a rotation around y-axis: $\beta=1.328^{\circ}$. We also observe that the displacement of the mirror platform center slightly varies from initial position at $2 \mathrm{~V}$ and the angle at this position is non-null as predicted. This fact can relate to the hypothesis stated during modeling process, which is only pure vertical displacement of the actuators occurs during tip-tilting motion. In reality, the horizontal component of each actuator's shifting is proven as not insignificant.

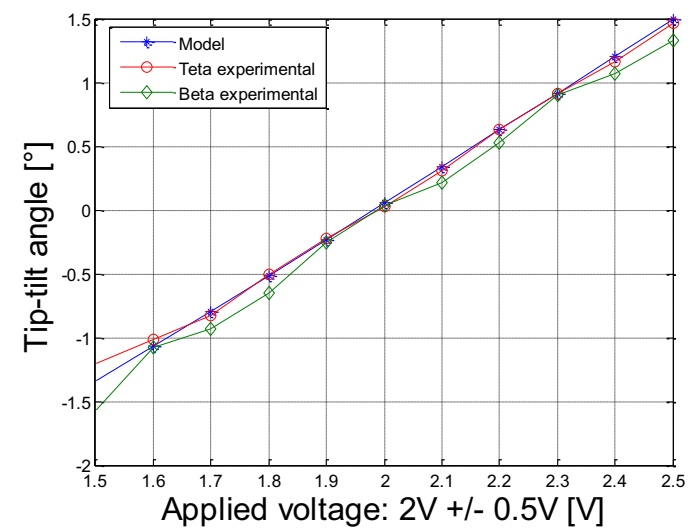

Figure 12 Comparison between each tip-tilt angle around an axis with the model

Both angle $\theta$ and $\beta$ are somewhat different which shows that the actuators are not identical. 


\subsubsection{DOF - Piston-Tip-Tilt motion}

We run this experiment using the parameters found in previous tests. In Fig. 13 both curves are nearly identical to model data.

The first try combines tip-tilt motion around $\mathrm{x}$-axis followed by another motion around $y$-axis. The maximum value of $\theta_{l}=1.463^{\circ}$ is fixed as the reference angle and we varied the driving voltages to obtain the value of $\beta_{2}$. Maximum angle achieved is $\beta_{2}=1.594^{\circ}$.

The same as in previous test, we switch the axis of tiptilt and the reference angle. This time, the tip-tilt motion is around $\mathrm{y}$-axis then followed by another motion around $\mathrm{x}$ axis. We fixed $\beta_{1}=1.328^{\circ}$ as reference and run the test to obtain the angle $\theta_{2}$. Maximum angle achieved is $\theta_{2}=1.359^{\circ}$. Once again, we observe the dissimilarity between the actuators, when we compare both experimental results between $\beta_{2}$ and $\theta_{2}$ for both cases.

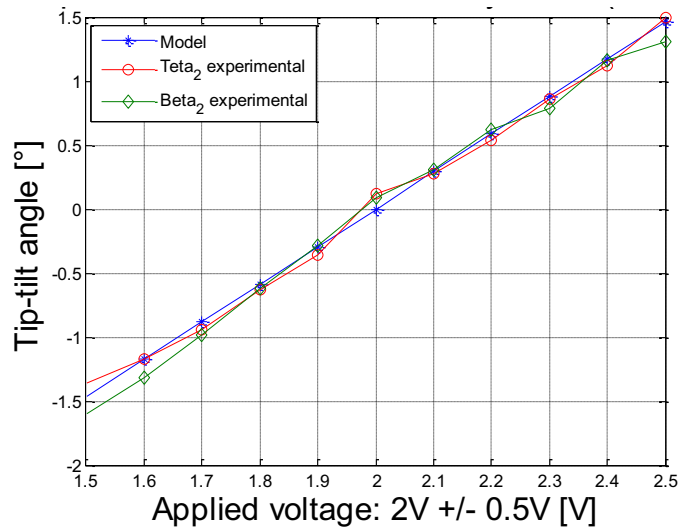

Figure 13 Comparison of 3 DoF tip-tilt angles with the model

\section{Discussion}

The static modeling and experimental results presented in this paper will help us to develop a dynamic control system for the MEMS mirror when performing the scanning.

The experimental setup will use ViSP and a high speed camera system. At first, we shall implement a closed-loop control to understand the behavior of the system. To do this, we will determine several scanning trajectories to scan the whole surface of the mirror. The trajectories will be transformed into series of coordinates that shall be compared with the actual coordinates given by ViSP. In operation it is desired to dynamically control the spot position on the screen, as in the OCT probe working principle.

The trajectory that allows the mirror to perform at low energy consumption will be chosen as well as a controller that will help to control the orientation of the mirror platform. This calibration step shall lead to developing an open loop control.

\section{Conclusion}

In this paper, a modeling of a $3 \mathrm{DoF}$ electrothermal actuated MEMS mirror based on parallel kinematics and multiphysical characteristics has been proposed. We have presented promising results on simulated and real data. We were also able to identify the physical aspects of the actuators. These results acknowledge the comprehension of the MEMS mirror mechanism which shows exceptional flexibility. We have also discussed an experimental setup for a control system for the MEMS mirror. We aim to implement a controller that shall be able to minimize the position error while scanning with precision at high frequency. This investigation is still in process.

\section{Acknowledgement}

These works were partly supported under the FrancheComte REGION, the Labex ACTION project and the French RENATECH network through its FEMTO-ST technological facility. The authors would like to acknowledge I. Anane and H. Nefzaoui for intermediary works.

\section{References}

[1] P.A. Himmer, D.L. Dickensheets, R.A. Friholm, Opt.Lett. 26 (16) (2001) 1280

[2] D.L. Polla, A.G. Erdman, W.P Robbins, D.T. Markus, J.Diaz-Diaz, R. Rizq, Y. Nam, H.T. Brickner, A. Wang, P. Krulevitch, Annu. Rev. Biomed. Eng 2 (2000) 551.

[3] J.M. Zara, S. Yazdanfar, K.D.Rao, J.A. Izzat, S.W. Smith, Opt. Lett. 28 (8) (2003) 628

[4] Y. Pan, H. Xie, G.K, Fedder, Opt. Lett. 26 (24) (2001) 1966.

[5] C. Chong, K. Isamoto, and H. Toshiyoshi, IEEE Photonics Technology Lett, vol. 10, pp. 570-578, (2006).

[6] K. Jia, S. Pal, and H. Xie, J. of MEMS, vol. 19, pp. 1004-1015, (2009).

[7] W. Liao, W. Liu, Y. Zhu, Y. Tang, B. Wang, and H. Xie, IEEE Sensors J., vol. 13, pp. 2873-2881, (2013).

[8] J. Singh, J. H. S. Teo, Y. Xu, C. S. Premachandran, N. Chen, R. Kotlanka, M. Olivo, and C. J. R. Sheppard, J. of Micromech \& Microeng., vol. 18, pp. 1-9, (2008)

[9] L. Liu, S. Pal, H. Xie, Sensors and Actuators A: Physical. 188, pp 349-358. (2012).

[10] L. Wu. H. Xie, "Sensors and Actuators A: Physics" 145-146. Pp. 371-379.(2008)

[11] K. Jia, S. Pal, and H. Xie, J. MEMS., vol. 18, no. 5, pp. 1004-1015, (2009).

[12] K. Jia, S.R. Samuelson, H. Xie, J. MEMS., vol. 20, no. 3, pp. 573 582, (2011).

[13] S. T. Todd, A. Jain, H. Qu and H. Xie, J. Opt. A, Pure Appl. Opt., vol. 8, no. 7, pp.S352 -S359, (2006)

[14] C. Clevy, M. Rakotondrabe, and N. Chaillet, Eds., Springer, (2011).

[15] K. Rabenorosoa, C. Clevy, S.Bargiel, J. P. Mascaro, P. Lutz, and C. Gorecki, J. of Micromechanics \& Microengineering, vol.20, pp. 045012, (2011).

[16] E. Pengwang, K. Rabenorosoa, M. Rakotondrabe, N. Andreff, (MESA), IEEE/ASME 10th International Conference, pp.1-6, (2014).

[17] A. Espinosa, K. Rabenorosoa, C. Clevy, B. Komati, P. Lutz, X. Zhang, S. R. Samuelson et H. Xie, International Journal of Optomechatronics, vol. 8, n 3, pp. 179-194, (2014). 